\title{
Edge Sum Number of Jahangir Graphs
}

\author{
${ }^{1}$ D. S. T. Ramesh, ${ }^{2}$ I. Gnanaselvi, ${ }^{3}$ S. Alice Pappa \\ ${ }^{I}$ Department of Mathematics, Margoschis College,Nazareth-628617, Tamil Nadu,India. \\ ${ }^{2}$ Department of Mathematics, Jayaraj Annapackiam CSI College of Engineering, Nazareth-628617,Tamil \\ Nadu,India. \\ ${ }^{3}$ Department of Mathematics, Margoschis College,Nazareth-628617, Tamil Nadu, India.
}

\begin{abstract}
A graph is said to be an edge sum graph if the edges of $G$ can be labeled with distinct positive integers such that the sum of all the labels incident on a vertex is again an edge label of $G$ and if the sum of any collection of edges is a label of an edge in $G$, then they are incident on a vertex. The edge sum number $\sigma_{\mathrm{E}}(\mathrm{G})$ of a graph $G$ is the smallest number $r$ of edges which added to $G$ result in an edge sum graph. In this paper, we prove that $\sigma_{\mathrm{E}}\left(\mathrm{J}_{3,4}\right)=3$.
\end{abstract}

Keywords: Sum graph, edge sum graph, sum number, edge sum number.

\section{Introduction}

All terms not defined here can be found in Harary [4]. Throughout this paper, we consider only finite undirected graphs without loops. By a graph $\mathrm{G}(\mathrm{V}, \mathrm{E})$ we mean a graph with vertex set $\mathrm{V}$ and edge set $\mathrm{E}$. Jahangir graphs $J_{n, m}$ for $m \geq 3$ is a graph on $n m+1$ vertices that is a graph consisting of a cycle $C_{n m}$ with one more vertex which is adjacent to $m$ vertices of $C_{n m}$ at distance $n$ to each on $C_{n m}$.

Harary [5] introduced the concept of sum graph and sum number. A graph $G$ is called a sum graph if the vertices of $\mathrm{G}$ can be labeled with distinct positive integers so that $\mathrm{e}=\mathrm{uv}$ is an edge of $\mathrm{G}$ if and only if the sum of the labels $u$ and $v$ equals a label of some vertex $w$ in $G$. If $G$ is not a sum graph, adding a finite number of isolated vertices to it always yields a sum graph and the sum number of $G$ is the smallest number of isolated vertices so added. T. Hao [3] proved an existence theorem for sum graphs and M. N. Elingham [2] proved that the sum number of any tree is just one. Several results on sum graphs and sum number of various graphs are $[1,6,7,10]$. D. S. T. Ramesh et. al. [8,9] defined edge sum graph, the edge analogue of sum graph and edge sum number which we denote by $\sigma_{\mathrm{E}}$. In this paper, we prove that $\sigma_{\mathrm{E}}\left(\mathrm{J}_{3,4}\right)=3$.

1.1 Definition Let $G(V, E)$ be a graph. Let $S$ be a set of positive integers. An edge labeling of $G$ by elements of $\mathrm{S}$ is a bijection $\mathrm{f}: \mathrm{E} \rightarrow \mathrm{S}$. It induces a vertex labeling $\mathrm{F}$ of positive integers defined by $\mathrm{F}(\mathrm{v})=\sum\{\mathrm{f}(\mathrm{e}): \mathrm{e}$ is incident on $\mathrm{v}\}$ for every $\mathrm{v} \in \mathrm{V}$. We call $\mathrm{f}$ an edge function of $\mathrm{G}$ and $\mathrm{F}$ an edge sum function of $\mathrm{G}$ induced by $\mathrm{f}$.

1.2 Definition $G$ is said to be an edge sum graph if there exists an edge function $f: E \rightarrow S$ such that $f$ and its corresponding edge sum function $F$ on $V$ satisfying the following conditions:

1. $\quad F$ is into $S$. That is, $F(v) \in S$ for every $v \in V$.

2. For any collection of edges $e_{1}, e_{2}, \ldots, e_{n} \in E$ such that $f\left(e_{1}\right)+f\left(e_{2}\right)+\ldots+f\left(e_{n}\right) \in S$, then $e_{1}, e_{2}, \ldots$ $\mathrm{e}_{\mathrm{n}}$ are incident on a vertex

1.3 Definition Let $G(V, E)$ be an edge sum graph. Let $e_{1}, e_{2}, \ldots, e_{m}$ where $m>1$ be a collection of edges incident on a vertex $\mathrm{w}$ (say). Let $\mathrm{ww}_{\mathrm{i}}=\mathrm{e}_{\mathrm{i}}$ for $1 \leq \mathrm{i} \leq \mathrm{m}$. If there exists an edge $\mathrm{e}=\mathrm{uv}$ such that $f\left(e_{1}\right)+f\left(e_{2}\right)+\ldots+f\left(e_{m}\right) \in f(e)$ and if $\operatorname{deg}(u) \geq 2$, then $u$ is adjacent to w. Similarly, for $v$.

Hence, if $F(w)=f(u v)$ and $\{u, v\}$ is not a $K_{2}$ component of $G$ then in $G$ either $\{u, v, w\}$ form a triangle or one of $\{\mathrm{u}, \mathrm{v}\}$ is adjacent to $\mathrm{w}$ and the other is a pendent vertex. 
1.4 Definition Let $\sigma_{E}(G)=r$. An edge function $f: E \rightarrow S$ and its corresponding edge sum function $F$ that makes $\mathrm{G} \cup \mathrm{rK}_{2}$ an edge sum graph are respectively called an optimal edge function and an optimal edge sum function of $\mathbf{G}$. For a graph $\mathrm{G}$ with $\sigma_{\mathrm{E}}(\mathrm{G})=\mathrm{r}$, there can be many optimal edge functions. Let $\mathrm{E}_{1}$ be the edge set of $G$ and $E_{2}$ be that of $\mathrm{rK}_{2}$. Then, $\sigma_{\mathrm{E}}(\mathrm{G})=$ Cardinality of the set $\left\{\mathrm{F}(\mathrm{v}): \mathrm{v} \in \mathrm{V} ; \mathrm{F}(\mathrm{v}) \notin \mathrm{f}\left(\mathrm{E}_{1}\right)\right\} . \mathrm{F}$ is said to be an outer edge sum function if $\mathrm{F}(\mathrm{V}) \cap \mathrm{f}\left(\mathrm{E}_{1}\right)=\phi$ and an inner edge sum function if $\mathrm{F}(\mathrm{V}) \cap \mathrm{f}\left(\mathrm{E}_{1}\right) \neq \phi$. The range of $\mathrm{F}$ has at least $\mathrm{r}$ elements. It has exactly $\mathrm{r}$ elements if and only if $\mathrm{F}$ is an outer edge sum function.

1.5 Theorem: Let $\mathrm{f}: \mathrm{E} \rightarrow \mathrm{S}$ be an optimal edge function. If $\mathrm{G}$ has no pendent vertex and is triangle free, then $\mathrm{F}$ is an outer edge sum function.

Proof: Let $E_{1}$ be the edge set of $G$ and $E_{2}$ that of $r_{2}$. Let $u \in V$. Since $F(u) \in S$, there is an edge vw such that $\mathrm{F}(\mathrm{u})=\mathrm{f}(\mathrm{vw})$. If $\mathrm{vw} \in \mathrm{E}_{1}$, then $\langle\mathrm{u}, \mathrm{v}, \mathrm{w}\rangle$ is $\mathrm{K}_{3}$ or $\mathrm{P}_{2}$ or $\mathrm{P}_{1}$ with $\mathrm{v}$ or $\mathrm{w}$ as a pendent vertex which is a contradiction. Hence $\mathrm{vw} \in \mathrm{E}_{2}$ so that $\mathrm{F}$ is an outer edge sum function.

4.2 Remark: It is easily seen that every optimal edge sum function $F$ of a graph $G$ is inner if $G$ has a pendent vertex and is outer if $G$ contains no pendent vertex and triangle free. If $G$ has no pendent vertex but contains a triangle then F can be either inner (See Figure 1.1(a)) or outer (See Figure 1.1(b)). Here we show that $\sigma_{\mathrm{E}}\left(\mathrm{W}_{4}\right)=1$.

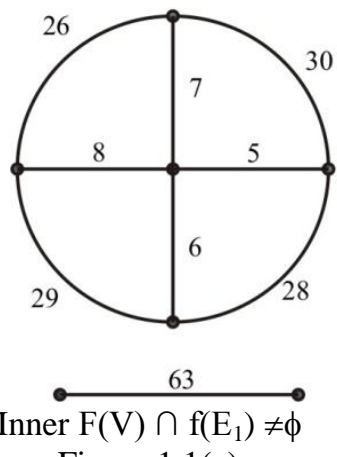

Figure 1.1(a)

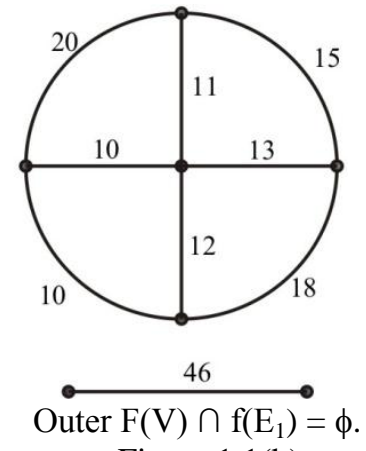

Figure 1.1(b)

\section{Edge Sum Number of Jahangir graphs}

2.1 Definition: Jahangir graphs $J_{n, m}$ for $m \geq 3$ is a graph on $n m+1$ vertices that is a graph consisting of a cycle $\mathrm{C}_{\mathrm{nm}}$ with one additional vertex which is adjacent to $\mathrm{m}$ vertices of $\mathrm{C}_{\mathrm{nm}}$ at distance $\mathrm{n}$ to each on $\mathrm{C}_{\mathrm{nm}}$.

2.1 Theorem $\sigma_{E}\left(J_{3,4}\right)=3$.

Proof: Let $\mathrm{G}=\mathrm{J}_{3,4}$ where $\mathrm{V}(\mathrm{G})=\{\mathrm{v}\} \cup\left\{\mathrm{v}_{\mathrm{i}, \mathrm{j}}: 1 \leq \mathrm{i} \leq 4 ; 1 \leq \mathrm{j} \leq 3\right\}$ and $\mathrm{E}(\mathrm{G})=$ $\left\{\mathrm{v}_{\mathrm{i}, \mathrm{j}} \mathrm{v}_{\mathrm{i}, \mathrm{j}+1}: 1 \leq \mathrm{i} \leq 4 ; 1 \leq \mathrm{j} \leq 2\right\} \cup\left\{\mathrm{v}_{\mathrm{i}, 3} \mathrm{v}_{\mathrm{i}+1,1}: 1 \leq \mathrm{i} \leq 3\right\} \cup\left\{\mathrm{v}_{4,3} \mathrm{v}_{1,1}\right\} \cup\left\{\mathrm{vv}_{\mathrm{i}, 1}: 1 \leq \mathrm{i} \leq 4\right\}$.

First let us prove that $\sigma_{\mathrm{E}}(\mathrm{G})>1$.

Suppose $\sigma_{\mathrm{E}}(\mathrm{G})=1$.

Then there exists an optimal edge function $\mathrm{f}$ and its corresponding edge sum function $\mathrm{F}$ such that $\mathrm{G} \cup \mathrm{K}_{2}$ is an edge sum graph. Let $\mathrm{w}_{1} \mathrm{w}_{2}$ be the $\mathrm{K}_{2}$ component of $\mathrm{G} \cup \mathrm{K}_{2}$. Since $\mathrm{G}$ is triangle free and has no pendent vertex; $\mathrm{F}$ is an outer edge sum function.

That is, $\mathrm{F}(\mathrm{u})=\mathrm{f}\left(\mathrm{w}_{1} \mathrm{w}_{2}\right)=\mathrm{a}$ (say) for all $\mathrm{u} \in \mathrm{V}$ 


$$
\begin{aligned}
& \mathrm{F}\left(\mathrm{v}_{1,2}\right) \quad=\mathrm{f}\left(\mathrm{v}_{1,1} \mathrm{v}_{1,2}\right)+\mathrm{f}\left(\mathrm{v}_{1,2} \mathrm{v}_{1,3}\right)=\mathrm{a} \\
& \mathrm{F}\left(\mathrm{v}_{1,3}\right) \quad=\mathrm{f}\left(\mathrm{v}_{1,2} \mathrm{v}_{1,3}\right)+\mathrm{f}\left(\mathrm{v}_{1,3} \mathrm{v}_{2,1}\right)=\mathrm{a}
\end{aligned}
$$

That is, $\mathrm{f}\left(\mathrm{v}_{1,1} \mathrm{v}_{1,3}\right)=\mathrm{f}\left(\mathrm{v}_{1,3} \mathrm{v}_{2,1}\right)$

This is not possible as $\mathrm{f}$ is a bijection. Hence $\sigma_{\mathrm{E}}(\mathrm{G})>1$.

Suppose $\sigma_{\mathrm{E}}(\mathrm{G})=2$.

Then there exists an optimal edge function $f$ and an optimal edge sum function $F$ such that $G \cup 2 K_{2}$ is an edge sum graph. Let $\mathrm{w}_{1} \mathrm{w}_{2}$ and $\mathrm{w}_{3} \mathrm{w}_{4}$ be the edges of the $\mathrm{K}_{2}$ component of $\mathrm{G} \cup 2 \mathrm{~K}_{2}$. Let $\mathrm{f}\left(\mathrm{w}_{1} \mathrm{w}_{2}\right)=\mathrm{z}$ and $\mathrm{f}\left(\mathrm{w}_{3} \mathrm{w}_{4}\right)=\mathrm{y} \quad$ where $\mathrm{z}=2 \mathrm{x}$.

Let $\mathrm{f}\left(\mathrm{v}_{1,1} \mathrm{v}_{1,2}\right)=\mathrm{x}-\mathrm{b}_{1} \Rightarrow \mathrm{f}\left(\mathrm{v}_{1,2} \mathrm{v}_{1,3}\right)=\mathrm{x}+\mathrm{b}_{1}$

$$
\begin{aligned}
& \mathrm{f}\left(\mathrm{v}_{1,2} \mathrm{v}_{1,3}\right)=\mathrm{x}+\mathrm{b}_{1} \Rightarrow \mathrm{f}\left(\mathrm{v}_{1,3} \mathrm{v}_{2,1}\right)=\mathrm{y}-\mathrm{x}-\mathrm{b}_{1} \\
& \mathrm{f}\left(\mathrm{v}_{2,2} \mathrm{v}_{2,3}\right)=\mathrm{x}-\mathrm{b}_{2} \Rightarrow \mathrm{f}\left(\mathrm{v}_{2,3} \mathrm{v}_{3,1}\right)=\mathrm{x}+\mathrm{b}_{2} \\
& \mathrm{f}\left(\mathrm{v}_{2,2} \mathrm{v}_{2,3}\right)=\mathrm{x}-\mathrm{b}_{2} \Rightarrow \mathrm{f}\left(\mathrm{v}_{2,1} \mathrm{v}_{2,2}\right)=\mathrm{y}-\mathrm{x}+\mathrm{b}_{2} \\
& \mathrm{f}\left(\mathrm{v}_{3,1} \mathrm{v}_{3,2}\right)=\mathrm{x}-\mathrm{b}_{3} \Rightarrow \mathrm{f}\left(\mathrm{v}_{3,2} \mathrm{v}_{3,3}\right)=\mathrm{x}+\mathrm{b}_{3} \\
& \mathrm{f}\left(\mathrm{v}_{3,2} \mathrm{v}_{3,3}\right)=\mathrm{x}+\mathrm{b}_{3} \Rightarrow \mathrm{f}\left(\mathrm{v}_{3,3} \mathrm{v}_{4,1}\right)=\mathrm{y}-\mathrm{x}-\mathrm{b}_{3} \\
& \mathrm{f}\left(\mathrm{v}_{4,2} \mathrm{v}_{4,3}\right)=\mathrm{x}-\mathrm{b}_{4} \Rightarrow \mathrm{f}\left(\mathrm{v}_{4,3} \mathrm{v}_{1,1}\right)=\mathrm{x}+\mathrm{b}_{4} \\
& \mathrm{f}\left(\mathrm{v}_{4,2} \mathrm{v}_{4,3}\right)=\mathrm{x}-\mathrm{b}_{4} \Rightarrow \mathrm{f}\left(\mathrm{v}_{4,1} \mathrm{v}_{4,2}\right)=\mathrm{y}-\mathrm{x}+\mathrm{b}_{4} \\
& \mathrm{Let} \mathrm{f}\left(\mathrm{vv}_{1,1}\right)=\mathrm{x}_{1} \\
& \mathrm{f}\left(\mathrm{vv}_{2,1}\right)=\mathrm{x}_{2} \\
& \mathrm{f}\left(\mathrm{vv}_{3,1}\right) \quad=\mathrm{x}_{3} \\
& \mathrm{f}\left(\mathrm{vv}_{4,1}\right)=\mathrm{x}_{4}
\end{aligned}
$$

Suppose

$$
\mathrm{F}\left(\mathrm{v}_{1,2}\right)=\mathrm{F}\left(\mathrm{v}_{2,1}\right)=\mathrm{F}\left(\mathrm{v}_{2,3}\right)=\mathrm{F}\left(\mathrm{v}_{3,2}\right)=\mathrm{F}\left(\mathrm{v}_{4,1}\right)=\mathrm{F}\left(\mathrm{v}_{4,3}\right)=2 \mathrm{x} \text { and }
$$

$$
\mathrm{F}\left(\mathrm{v}_{1,1}\right)=\mathrm{F}\left(\mathrm{v}_{1,3}\right)=\mathrm{F}\left(\mathrm{v}_{2,2}\right)=\mathrm{F}\left(\mathrm{v}_{3,1}\right)=\mathrm{F}\left(\mathrm{v}_{3,3}\right)=\mathrm{F}\left(\mathrm{v}_{4,2}\right)=\mathrm{y}
$$$$
\mathrm{F}\left(\mathrm{v}_{1,1}\right)=\mathrm{f}\left(\mathrm{vv}_{1,1}\right)+\mathrm{f}\left(\mathrm{v}_{4,3} \mathrm{v}_{1,1}\right)+\mathrm{f}\left(\mathrm{v}_{1,1} \mathrm{v}_{1,2}\right)=\mathrm{y}
$$

$$
\Rightarrow \mathrm{x}_{1}+2 \mathrm{x}-\mathrm{b}_{1}+\mathrm{b}_{4}=\mathrm{y}
$$$$
\Rightarrow \mathrm{x}_{1}=\mathrm{y}-2 \mathrm{x}+\mathrm{b}_{1}-\mathrm{b}_{4}
$$

$$
\begin{gathered}
\mathrm{F}\left(\mathrm{v}_{2,1}\right)=\mathrm{f}\left(\mathrm{vv}_{2,1}\right)+\mathrm{f}\left(\mathrm{v}_{1,3} \mathrm{v}_{2,1}\right)+\left(\mathrm{v}_{2,1} \mathrm{v}_{2,2}\right)=2 \mathrm{x} \\
\Rightarrow \mathrm{x}_{2}+2 \mathrm{y}-2 \mathrm{x}-\mathrm{b}_{1}+\mathrm{b}_{2}=2 \mathrm{x}
\end{gathered}
$$$$
\Rightarrow \mathrm{x}_{2}=4 \mathrm{x}-2 \mathrm{y}+\mathrm{b}_{1}-\mathrm{b}_{2}
$$

$$
\mathrm{F}\left(\mathrm{v}_{3,1}\right)=\mathrm{f}\left(\mathrm{vv}_{3,1}\right)+\mathrm{f}\left(\mathrm{v}_{2,3} \mathrm{v}_{3,1}\right)+\mathrm{f}\left(\mathrm{v}_{3,1} \mathrm{v}_{3,2}\right)=\mathrm{y}
$$$$
\Rightarrow \mathrm{x}_{3}+2 \mathrm{x}+\mathrm{b}_{2}-\mathrm{b}_{3}=\mathrm{y}
$$$$
\Rightarrow \mathrm{x}_{3}=\mathrm{y}-2 \mathrm{x}+\mathrm{b}_{3}-\mathrm{b}_{2}
$$

$$
\begin{gathered}
\mathrm{F}\left(\mathrm{v}_{4,1}\right)=\mathrm{f}\left(\mathrm{vv}_{4,1}\right)+\mathrm{f}\left(\mathrm{v}_{3,3} \mathrm{v}_{4,1}\right)+\mathrm{f}\left(\mathrm{v}_{4,1} \mathrm{v}_{4,2}\right)=2 \mathrm{x} \\
\Rightarrow \mathrm{x}_{4}+2 \mathrm{y}-2 \mathrm{x}-\mathrm{b}_{3}+\mathrm{b}_{4}=2 \mathrm{x}
\end{gathered}
$$




$$
\begin{aligned}
& \Rightarrow \mathrm{x}_{4}=4 \mathrm{x}-2 \mathrm{y}+\mathrm{b}_{3}-\mathrm{b}_{4} \\
& \mathrm{~F}(\mathrm{v})=\mathrm{f}\left(\mathrm{vv}_{1,1}\right)+\mathrm{f}\left(\mathrm{vv}_{2,1}\right)+\mathrm{f}\left(\mathrm{vv}_{3,1}\right)+\mathrm{f}\left(\mathrm{vv}_{4,1}\right) \\
& =\mathrm{x}_{1}+\mathrm{x}_{2}+\mathrm{x}_{3}+\mathrm{x}_{4} \\
& \Rightarrow b_{1}-b_{4}>a \\
& \mathrm{x}_{3}=\mathrm{b}_{3}-\mathrm{b}_{2}-\mathrm{a} \Rightarrow \mathrm{b}_{3}>\mathrm{a}+\mathrm{b}_{2} \\
& \Rightarrow \mathrm{b}_{3}-\mathrm{b}_{2}>\mathrm{a} \\
& \mathrm{x}_{2}=2 \mathrm{a}+\mathrm{b}_{1}-\mathrm{b}_{2} \\
& \mathrm{x}_{4}=2 \mathrm{a}+\mathrm{b}_{3}-\mathrm{b}_{4} \\
& \mathrm{x}_{2}+\mathrm{x}_{4}=4 \mathrm{a}+\mathrm{b}_{1}-\mathrm{b}_{2}+\mathrm{b}_{3}-\mathrm{b}_{4} \\
& =4 a+b_{1}-b_{4}+b_{3}-b_{2} \\
& >4 a+a+a=6 a \\
& \mathrm{x}_{1}+\mathrm{x}_{2}+\mathrm{x}_{3}+\mathrm{x}_{4}>\mathrm{x}_{1}+\mathrm{x}_{3}+6 \mathrm{a} \\
& >\mathrm{x}_{1}+\mathrm{x}_{3}+12 \mathrm{x} \text { (since } \mathrm{a}>2 \mathrm{x} \text { ) } \\
& >12 \mathrm{x}
\end{aligned}
$$

This is a contradiction.

Case (ii)

If $F(v)=2 x$ where $2 x<y$

Let $y-2 x=b$

Therefore $\mathrm{b}>\mathrm{y}>2 \mathrm{x}$

$$
\begin{aligned}
& \mathrm{x}_{1}=\mathrm{b}+\mathrm{b}_{1}-\mathrm{b}_{4} \\
& \mathrm{x}_{3}=\mathrm{b}+\mathrm{b}_{3}-\mathrm{b}_{2} \\
& \mathrm{x}_{2}=\mathrm{b}_{1}-\mathrm{b}_{2}-2 \mathrm{~b} \\
& \Rightarrow \mathrm{b}_{1}-\mathrm{b}_{2}>2 \mathrm{~b} \\
& \mathrm{x}_{4}=\mathrm{b}_{3}-\mathrm{b}_{4}-2 \mathrm{~b} \\
& \Rightarrow \mathrm{b}_{3}-\mathrm{b}_{4}>2 \mathrm{~b} \\
& \mathrm{x}_{1}+\mathrm{x}_{3}=2 \mathrm{~b}+\mathrm{b}_{1}-\mathrm{b}_{2}+\mathrm{b}_{3}-\mathrm{b}_{4} \\
& >2 b+2 b+2 b \\
& =6 b>y>2 \mathrm{x}
\end{aligned}
$$

This is a contradiction.

$$
\mathrm{y} \geq \mathrm{x}_{1}+\mathrm{x}_{2}+\mathrm{x}_{3}+\mathrm{x}_{4}>\mathrm{x}_{1}+\mathrm{x}_{3}>6 \mathrm{~b}>\mathrm{y}
$$

Hence $\sigma_{\mathrm{E}}(\mathrm{G})>2$.

The edge function given in Figure 2.1 shows that $\sigma_{\mathrm{E}}\left(\mathrm{J}_{3,4}\right)=3$. 


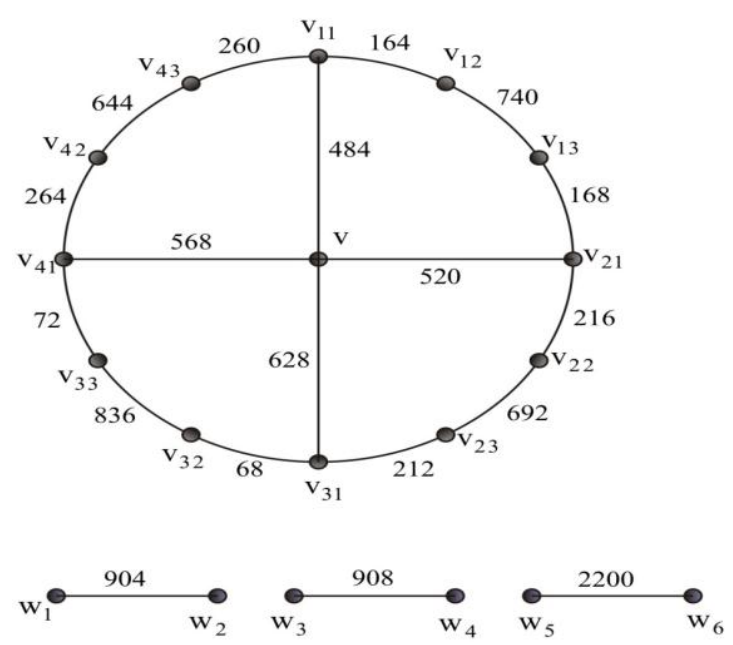

Figure 2.1

\section{References}

[1] D. Berstrnd, F. Harary et. al. "The Sum number of a complete graph”, Bull. Malaysian Math. Soc., (1989) $25-28$.

[2] M. N. Elligham, "Sum graphs from trees", Ars Comb. 35 (1993), 335-349.

[3] T. Hao, “On sum graphs", J. Comb. Math. Combin. Comput. 6 (1989), 207-212.

[4] F. Harary, Graph Theory, Addison-Wesley, Reading, Mass. (1968).

[5] F. Harary, "Sum graphs and difference graphs", Cong. Number., 72, (1990), 101-108.

[6] M. Miller et. al., "The sum number of the covktail party graph", Bulletin of the Institute of Combinatorics and its applications, vol. 22 (1998), 79-90.

[7] T. Nicholas, S. Somasundaram and Vilfred, "Some results on sum graphs", Journal of Combinatorics and system sciences, vol. 26, Nos. 1-4 (2001), 135-142.

[8] D. S. T. Ramesh, J. Paulraj Joseph and S. Somasundaram, "Edge Sum Graphs", InternationalJournal of Management \& Systems, vol 18, No. 1, (2002), 71-78.

[9] D. S. T. Ramesh, J. Paulraj Joseph and S. Somasundaram, "Edge Sum Number of Graphs", InternationalJournal of Management \& Systems, vol 19, No. 1, (2003), 25-36.

[10] W. F. Smyth, "Sum graphs of small number”, Colloquia Math. Soc. Janos Bolyai, 60 (1991), 669-678. 